\title{
A tabletop setup for ultrafast helicity- dependent and element-specific absorption spectroscopy and scattering in the extreme ultraviolet spectral range ${ }^{\circledR}$
}

Cite as: Rev. Sci. Instrum. 91, 093001 (2020); https://doi.org/10.1063/5.0013928

Submitted: 15 May 2020. Accepted: 05 September 2020 . Published Online: 23 September 2020

Kelvin Yao, (D) Felix Willems, (D) Clemens von Korff Schmising, (D) Christian Strüber, Piet Hessing, (D) Bastian Pfau, (iD) Daniel Schick, (D) Dieter Engel, (D) Kathinka Gerlinger, (iD Michael Schneider, and Stefan Eisebitt

\section{COLLECTIONS}

EP This paper was selected as an Editor's Pick
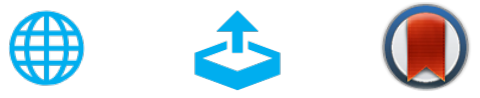

\section{ARTICLES YOU MAY BE INTERESTED IN}

A strip-type boron-doped diamond heater synthesized by chemical vapor deposition for large-volume presses

Review of Scientific Instruments 91, 095108 (2020); https://doi.org/10.1063/5.0011742

Materials loss measurements using superconducting microwave resonators

Review of Scientific Instruments 91, 091101 (2020); https://doi.org/10.1063/5.0017378

Influence of pressure transducer protrusion depth on pressure measurements of shock waves in shock tubes

Review of Scientific Instruments 91, 106101 (2020); https://doi.org/10.1063/5.0016593

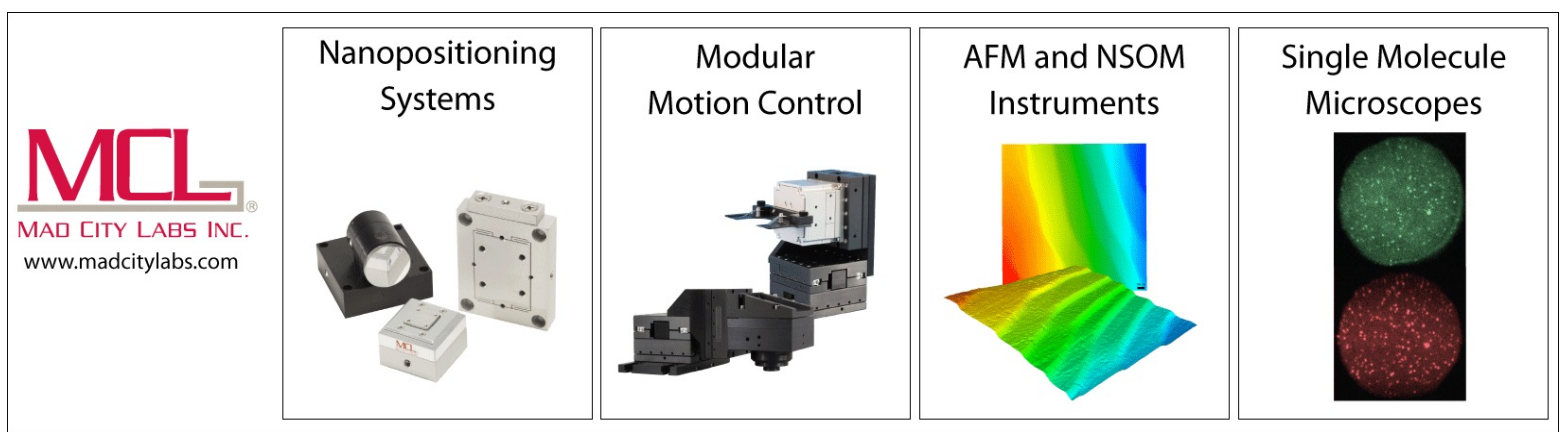




\title{
A tabletop setup for ultrafast helicity-dependent and element-specific absorption spectroscopy and scattering in the extreme ultraviolet spectral range ${ }^{\circ}$
}

\author{
Cite as: Rev. Sci. Instrum. 91, 093001 (2020); doi: 10.1063/5.0013928 \\ Submitted: 15 May 2020 - Accepted: 5 September 2020 • \\ Published Online: 23 September 2020
}

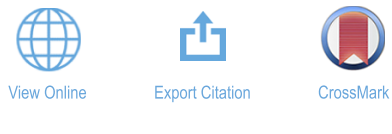

Kelvin Yao, ${ }^{1}$ Felix Willems, , ,a) (D) Clemens von Korff Schmising, ${ }^{1, a)}$ (D) Christian Strüber, ${ }^{1, b)}$ (D) Piet Hessing,

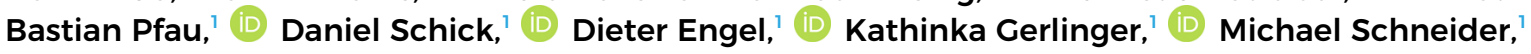
and Stefan Eisebitt ${ }^{1,2}$

\author{
AFFILIATIONS \\ ${ }^{1}$ Max-Born-Institut für Nichtlineare Optik und Kurzzeitspektroskopie, Max-Born-Straße 2A, 12489 Berlin, Germany \\ ${ }^{2}$ Technische Universität Berlin, Institut für Optik und Atomare Physik, 10623 Berlin, Germany
}

a) Authors to whom correspondence should be addressed:willems@mbi-berlin.de and korff@mbi-berlin.de

b) Present address: Department of Physics, Free University Berlin, Arnimallee 14, 14195 Berlin, Germany.

\begin{abstract}
Further advances in the field of ultrafast magnetization dynamics require experimental tools to measure the spin and electron dynamics with element-specificity and femtosecond temporal resolution. We present a new laboratory setup for two complementary experiments with light in the extreme ultraviolet (XUV) spectral range. One experiment is designed for polarization-dependent transient spectroscopy, particularly for simultaneous measurements of magnetic circular dichroism (MCD) at the $3 p$ resonances of the $3 d$ transition metals Fe, Co, and Ni. The second instrument is designed for resonant small-angle scattering experiments with monochromatic light allowing us to monitor spin dynamics with spatial information on the nanometer scale. We combine a high harmonic generation (HHG) source with a phase shifter to obtain XUV pulses with variable polarization and a flux of about $(3 \pm 1) \times 10^{10}$ photons/s/harmonic at $60 \mathrm{eV}$ at the source. A dedicated reference spectrometer effectively reduces the intensity fluctuations of the HHG spectrum to below $0.12 \%$ rms. We demonstrate the capabilities of the setup by capturing the energy- and polarization-dependent absorption of a thin Co film as well as the time-resolved small-angle scattering in a magnetic-domain network of a Co/Pt multilayer. The new laboratory setup allows systematic studies of optically induced spin and electron dynamics with element-specificity, particularly with MCD as the contrast mechanism with femtosecond temporal resolution and an unprecedented signal-to-noise ratio.
\end{abstract}

Published under license by AIP Publishing. https://doi.org/10.1063/5.0013928

\section{INTRODUCTION}

Ultrafast magnetism belongs to the most active research fields in solid-state physics. Since the discovery of optically induced subpicosecond quenching of the magnetic moment, ${ }^{1}$ intensive research has been conducted to understand the microscopic mechanisms leading to ultrafast spin dynamics. Despite many observations of intriguing magnetic effects, particularly in multi-elemental compounds, such as all-optical switching, ${ }^{2,3}$ spintronic $\mathrm{THz}$ emission, ${ }^{4}$ or antiferromagnetic to ferromagnetic phase transitions via intersite spin transfer, ${ }^{6}$ a comprehensive fundamental understanding still does not exist. Generally, the optically induced functionality of magnetic materials depends on the individual contributions of its subsystems, which makes their disentanglement essential for a fundamental understanding.

To gain element-specific access to the spin dynamics, experimental techniques, such as time-resolved magnetic circular dichroism (MCD) spectroscopy, have been pioneered at large-scale facilities, in particular, at slicing beamlines using synchrotron radiation $^{7,8}$ and at free-electron lasers operating at $L^{-}{ }^{9}$ and $M$ absorption edges $^{10}$ of the $3 d$ transition metals (TMs). MCD describes the helicity-dependent absorption of resonant radiation in 
magnetic materials and is proportional to the magnetization of the material.

The continuously developing technology of intense short-pulse lasers and high harmonic generation (HHG) made it possible to transfer femtosecond extreme ultraviolet (XUV) spectroscopy experiments to laboratories. ${ }^{11-13}$ The most prominent geometry for HHG experiments until today exploits the transverse magnetooptical Kerr effect (T-MOKE), where the incident linearly polarized XUV light is reflected off the magnetic sample perpendicularly to the magnetic field direction. ${ }^{14,15}$ T-MOKE has gained popularity for two main reasons. First, it does not require additional control of the XUV polarization state, and second, the method offers high magnetic contrast of up to $90 \%$ in $\mathrm{Fe},{ }^{15,16}$ which allows obtaining sufficiently high signal-to-noise ratios even with highly fluctuating spectral intensities from HHG sources. In contrast, MCD spectroscopy requires circular polarization and suffers from signal levels typically one order of magnitude smaller than in T-MOKE experiments. This additional complexity might be the reason why the literature reports on HHG applications of MCD spectroscopy are sparse. ${ }^{13,17}$

However, MCD offers significant advantages. MCD in transmission is not limited to surface measurements, which pose inherent challenges, such as photon energy-dependent penetration depths. Unlike T-MOKE in reflection geometry and Faraday rotation in transmission geometry, ${ }^{18} \mathrm{MCD}$ can be described almost entirely by the absorptive part of the complex refractive index. ${ }^{19}$ This fact simplifies the interpretation of the data and allows, for example, monitoring the spin-resolved empty states around the Fermi level via helicity-dependent absorption spectroscopy. ${ }^{20}$ Furthermore, XUV MCD in transmission is particularly suitable for the simultaneous measurement of individual spin dynamics in multi-element samples because the absorptive part has a much narrower element-specific signature than the dispersive part. This is particularly important in the study of the $3 d$ TMs, as their $M$-absorption edges are spectrally very close to each other.
In this review, we present a new HHG laboratory setup consisting of two complementing beamlines. One beamline is designed for time-resolved and polarization-dependent magnetic absorption spectroscopy, allowing for energy-resolved MCD experiments. The other beamline is a versatile scattering instrument for resonant magnetic small-angle scattering and imaging experiments with nanometer spatial and femtosecond temporal resolution. A HHG source provides a broad spectrum of discrete harmonics with pulse lengths well below 25 fs and a flux of $\sim(3 \pm 1) \times 10^{10}$ photons/s/harmonic at $60 \mathrm{eV}$. We use a four-mirror reflection XUV phase shifter ${ }^{13,21}$ to control the polarization state between linear and circular in both beamlines. In the spectroscopy beamline, we use a dedicated reference spectrometer to normalize the HHG-typical intensity fluctuations. This normalization allows us to measure signal changes in the per mille range in the helicity-dependent absorption with an unprecedented signal-to-noise ratio.

In Sec. II, we first give an overview of the entire setup and then discuss the properties of the two beamlines. Subsection II A describes the features of the XUV radiation generation process. In Subsection II B, we describe details of the different parts in the spectroscopy beamline, in particular, the XUV polarizer and the reference spectrometer used for XUV intensity normalization. In Subsections II C and II D, we explain details and present results of time-resolved measurements in the spectroscopy and scattering beamlines, respectively. The results show the ultrafast magnetization dynamics measured with XUV MCD on a thin Co layer and with XUV small-angle scattering on a $\mathrm{Co} / \mathrm{Pt}$ multilayer and demonstrate the performance of our setup to resolve even smallest magnetic signals and their optically induced changes.

\section{EXPERIMENTAL SETUP}

Figure 1 depicts a schematic overview of the HHG setup consisting of a beamline for helicity-dependent spectroscopy and a

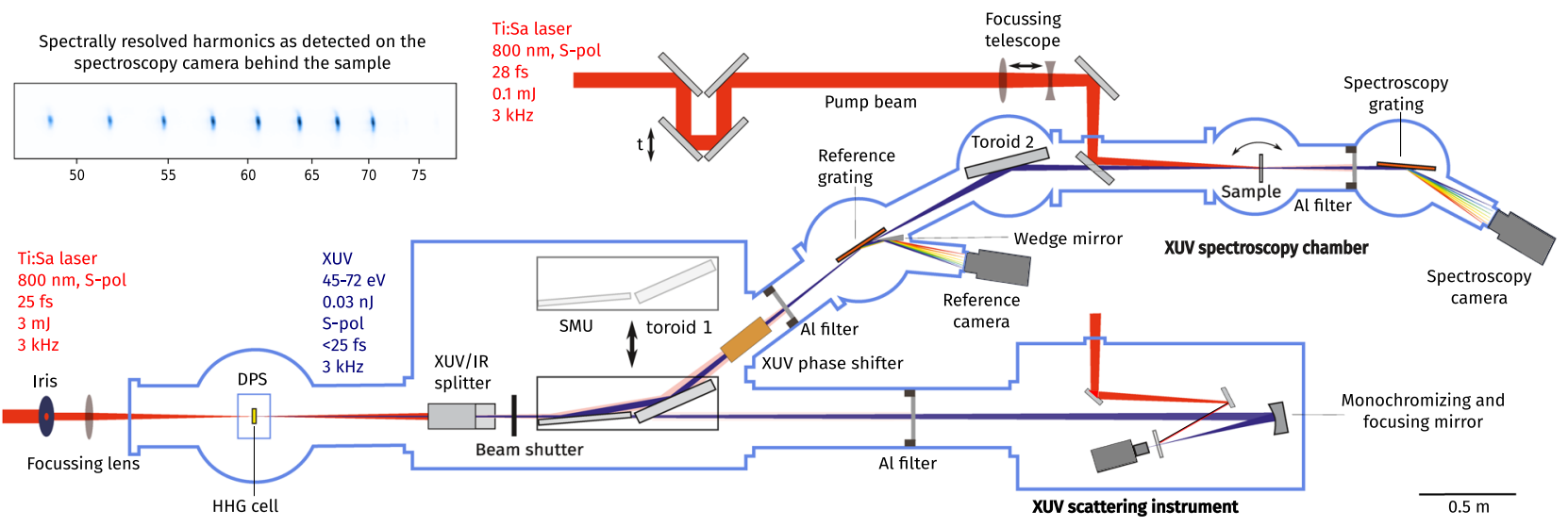

FIG. 1. Schematic drawing of the setup. The blue line encircles the components in vacuum with pressures $<10^{-6}$ mbar. High harmonics are generated in the HHG cell by intense IR pulses, which are consequently removed via the XUV/IR splitter and Al filters. The switching mirror unit (SMU) directs the XUV radiation to the spectroscopy beamline or, if removed, lets the beam pass into the scattering instrument. The reference grating and camera are used for intensity normalization. The pump beam is collimated by a telescope and enters the vacuum chamber about $900 \mathrm{~mm}$ before the sample, from where the pump and probe beams propagate nearly co-linearly (angle $\approx 0.3^{\circ}$ ). The inset in the upper left shows an image of the high harmonic spectrum recorded with the spectrometer behind the sample. 
beamline for small-angle scattering experiments. The parts within the blue contour are under high vacuum with a base pressure of below $10^{-6}$ mbar. Both experiments share the same HHG source; a motorized switching mirror unit (SMU) mounted at a grazing incidence angle of $4^{\circ}$ allows a rapid and reproducible redirection of the $\mathrm{XUV}$ radiation. In the spectroscopy beamline, a set of two identical toroidal mirrors focus the beam first onto a reference spectrometer and then onto the sample. The geometry of the focusing optics is determined by a long radius $R_{\text {tang }}=-11473 \mathrm{~mm}$ and a short radius $R_{\text {sag }}=-87 \mathrm{~mm}$, leading to a grazing incidence angle of $5^{\circ}$ and a focal length of $f=1000 \mathrm{~mm}$. Both toroidal mirrors are coated with a 20 -nm boron carbide $\left(\mathrm{B}_{4} \mathrm{C}\right)$ layer for broadband reflectance up to the carbon $K$-edge. After transmission through the sample, the XUV radiation is detected by a second spectrometer. In time-resolved experiments, we use a small fraction of the fundamental infrared (IR) pulse for optical excitation. The beam pointing of pump and probe pulses is actively stabilized via two motorized mirrors and detector pairs. For the probe beam, we use a commercial system working at a $200 \mathrm{~Hz}$ repetition rate (Compact, MRC Systems GmbH, Germany), while pointing instabilities of the pump beam due to imperfections of the mechanical delay line are compensated by a system, which we built ourselves. The grating and detector assembly can be rotated around the sample position and positioned at fixed angles $\theta=8^{\circ}$, $24^{\circ}$, and $35^{\circ}$ to also allow experiments in reflection, e.g., T-MOKE geometry. The Python-based supervisory control and data acquisition system Sardana ${ }^{22}$ is used for experimental control, allowing for highly customizable macro-based automated measurement routines.

\section{A. XUV generation}

The XUV radiation is generated via a high harmonic generation process, which is driven by a commercial Ti:sapphire regenerative laser amplifier (Legend Elite Duo HE + USX, Coherent Inc., USA): center wavelength $\lambda \approx 800 \mathrm{~nm}$ with a bandwidth of $80 \mathrm{~nm}$, a pulse duration of $25 \mathrm{fs}$ [full width at half maximum, (FWHM)], a pulse energy of $3 \mathrm{~mJ}$, a repetition rate of $3 \mathrm{kHz}$, and a beam diameter of $12 \mathrm{~mm}$ (FWHM). The pulses are focused by a lens $(f=1 \mathrm{~m})$ into a 2 - $\mathrm{mm}$ long gas cell containing the noble gases $\mathrm{He}$ or Ne for converting a fraction of the fundamental beam into a multi-color pulse train of odd high harmonics with an energy spacing of $\sim 3.1 \mathrm{eV}$. A differential pumping station (DPS) inside the HHG vacuum chamber enables gas pressures of over 1 bar inside the gas cell while preserving the chamber pressure on the order of $10^{-5}$ mbar.

Figure 2 shows the typical spectra and photon numbers measured with the spectrometer behind the sample. The orange curve in Fig. 2 shows the spectrum at the sample position with a photon count of $(6.0 \pm 1.2) \times 10^{9}$ photons/s integrated over the eight harmonic emission peaks in the energy range between $45 \mathrm{eV}$ and $72 \mathrm{eV}$. The blue curve represents the total flux calculated back to the source and amounts to $(2.7 \pm 0.9) \times 10^{11}$ photons/s. Each pulse carries an energy of $(0.8 \pm 0.3) \mathrm{nJ}$, calculated from the eight harmonics in the spectrum and their respective energies. The bandwidth of one harmonic is $\sim 200 \mathrm{meV}$, and the divergence of the XUV beam is $\sim 2 \mathrm{mrad}$ at $60 \mathrm{eV}$.

To obtain this spectrum and flux, the HHG process was optimized by tuning three parameters: pulse length, wavefront shape, and gas pressure, which can be controlled by a motorized

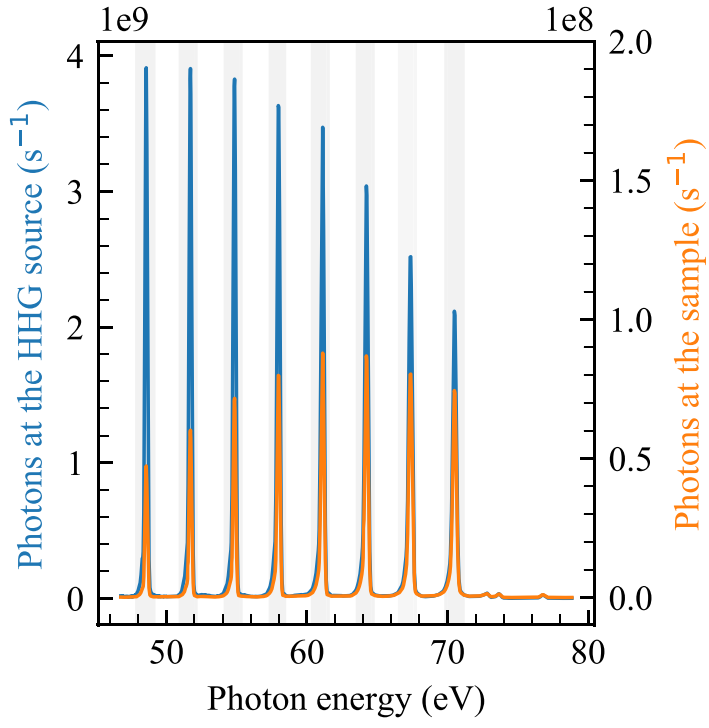

FIG. 2. The orange line shows the HHG spectrum at the sample position in the spectroscopy arm with a total photon count of $(6.0 \pm 1.2) \times 10^{9} / \mathrm{s}$, integrated over the light gray shaded area of all eight harmonic peaks in the energy range from $45 \mathrm{eV}$ to $72 \mathrm{eV}$. The blue line shows the HHG spectrum calculated back to the source with a flux of $(2.7 \pm 0.9) \times 10^{11}$ photons $/ \mathrm{s}$. We used a $2-\mathrm{mm}$ long gas cell containing Ne with a backing pressure of $230 \mathrm{mbar}$ and an iris size of $10 \mathrm{~mm}$. The spectrum is cut off by the $\mathrm{Al} L_{3}$-absorption edge at $72.55 \mathrm{eV}$.

compressor grating, a motorized iris diaphragm, and a mass flow controller (SLA5800 Series, Brooks Instruments, USA), respectively. The infrared (IR) beam shape and intensity are tuned by partially closing the iris diaphragm in front of the focusing lens. Closing the iris blocks large transverse propagation vectors from the beam, thus, shaping the wavefront at the focus of the beam in the HHG cell. ${ }^{24}$ We find the optimum aperture diameter at about $10 \mathrm{~mm}$ for $\mathrm{Ne}$ and $12.5 \mathrm{~mm}$ for He.

Figure 3 shows the HHG flux for the high harmonic peak around $60 \mathrm{eV}$ generated in $\mathrm{Ne}$ and $\mathrm{He}$ as a function of the backing gas pressure in the HHG cell. The optimum pressure in this setup is around $300 \mathrm{mbar}$ for $\mathrm{Ne}$ and $800 \mathrm{mbar}$ for He. At pressures above the optimum, the generated XUV is reabsorbed by gas atoms in the beam path, leading to a flux reduction. The maximum flux reaches $(3.3 \pm 1.2) \times 10^{10}$ photons/s/harmonic at $60 \mathrm{eV}$ corresponding to a conversion efficiency for the individual harmonic of (1.1 $\pm 0.4) \times 10^{-9}$. Note that Ne provides a higher XUV yield than $\mathrm{He}$ in the relevant energy range between $45 \mathrm{eV}$ and $72.55 \mathrm{eV}^{25,26} \mathrm{lim}$ ited by the $\mathrm{Al} L_{3}$-absorption edge. ${ }^{23}$ Details on the determination of the absolute number of generated XUV photons are given in the Appendix.

\section{B. Beamline layout}

\section{The XUV/IR beam splitter}

A challenge in experiments with high harmonic radiation is the separation of the XUV from the co-propagating and intense IR light. We use an XUV/IR beam splitter to first attenuate the IR-radiation by $2-3$ orders of magnitude and finally remove the remaining IR 


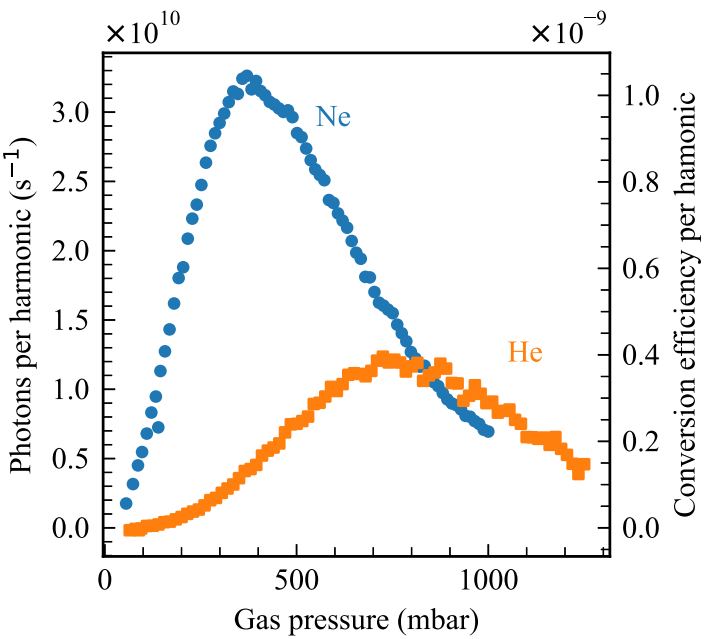

FIG. 3. Generated XUV photons per harmonic per second at $60 \mathrm{eV}$ and its conversion efficiency from the fundamental IR beam as a function of the gas pressure for helium and neon.

and visible light with a $200-\mathrm{nm}$ thin $\mathrm{Al}$ foil mounted in a removable vacuum gate valve. This two-stage separation prevents the IR beam from destroying the thin $\mathrm{Al}$ filter. On the other hand, the attenuated IR beam can be used for further alignment of the XUV optics when the Al filter is removed from the beam path. The XUV/IR splitter consists of a pair of parallel quartz glass substrates with a tailored $\mathrm{Si} / \mathrm{B}_{4} \mathrm{C}$ coating of $51 \mathrm{~nm} / 3 \mathrm{~nm}$ thickness, mounted close to its grazing Brewster angle of $\theta_{\mathrm{B}}=7.2^{\circ}$ for $\lambda=800 \mathrm{~nm}$. The splitter shifts the beam height, with the mirror surface being perpendicular to the other mirror planes so that the polarization and reflection planes of the mirror are in $p$-polarization geometry.

Figure 4(a) shows a side view sketch of the XUV/IR splitter and the calculated reflection of perfectly $p$-polarized light $R_{p}(\theta)$ for $1.55 \mathrm{eV}$ (IR) and $60 \mathrm{eV}$ (XUV) as a function of the angle of incidence $\Phi$. We calculated the values with optical constants tabulated in Refs. 27-29. The coating design is chosen to shift the Brewster angle to a minimal grazing angle of only $\theta_{\mathrm{B}} \approx 7^{\circ}$ compared to $\approx 15^{\circ}$ for bulk silicon. This design significantly increases the XUV reflectance of the XUV/IR beam splitter, resulting in a total transmission through the device of $\sim 60 \%$. An additional advantage compared to bulk $\mathrm{Si}$ is that only a negligible amount of the $800 \mathrm{~nm}$ light is absorbed, reducing heat-induced deformations of the mirror surface.

\section{The XUV phase shifter}

To generate circularly polarized XUV light, we built a miniaturized copy of a four-mirror reflection phase shifter, as presented in Ref. 21 and installed at the free-electron laser facility FLASH I at DESY, Germany.

Figure 4(b) shows a side view sketch of the phase shifter and its performance, i.e., the degree of ellipticity $P$ and the transmittance $T$ as a function of the photon energy. ${ }^{21}$ The data points represent the measured values, and the solid lines represent the calculated values.

We determined that a Mo $(20 \mathrm{~nm}) / \mathrm{B}_{4} \mathrm{C}(2 \mathrm{~nm})$ mirror coating for a fixed angle of grazing incidence of $12^{\circ}$ offers the most advantageous trade-off between $T$ and $P$, maximizing the figure of
A) XUV/IR splitter
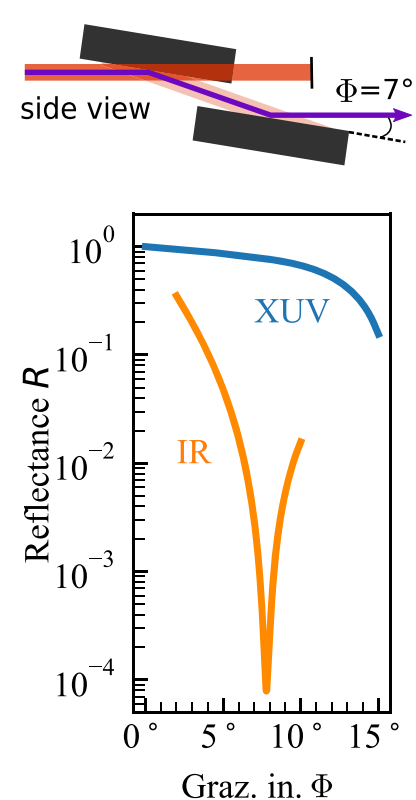

B) XUV phase shifter
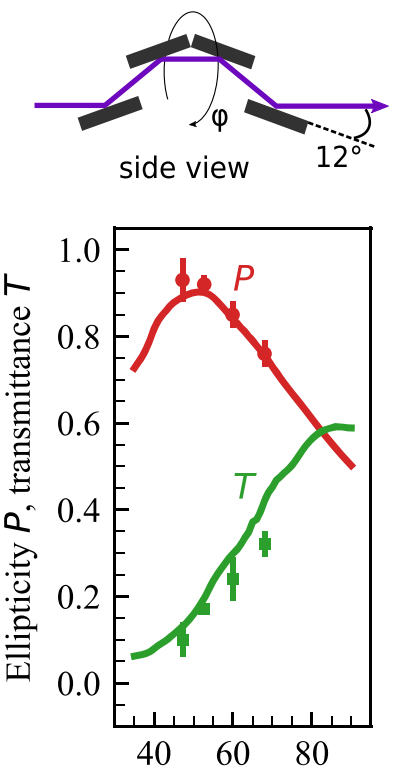

Photon energy $(\mathrm{eV})$
FIG. 4. (a) Reflectance of the XUV/IR splitter calculated for $1.55 \mathrm{eV}$ and $60 \mathrm{eV}$ as a function of the grazing incidence angle $(\Phi)$. (b) Transmittance $T$ and ellipticity $P$ of the four-mirror reflection phase shifter for photon energies between $40 \mathrm{eV}$ and $70 \mathrm{eV}$ at a fixed angle of grazing incidence of $12^{\circ}$. The data points represent the measured values, and the solid lines represent the calculated values.

merit $T P^{2}$. This configuration yields a broadband ellipticity of up to $80 \%$ and transmission of up to $30 \%$. Higher degrees of ellipticity can be achieved for larger angles of grazing incidence at the cost of a significantly reduced transmission.

The ratio between $s$ - and $p$-polarization incident on the mirror surfaces determines the phase shift of the XUV light and can be controlled by rotating the four-mirror unit around the beam axis $(\phi)$. Turning the polarizer from $0^{\circ}$ to $\pm 48^{\circ}$ changes the polarization from linear to right and left circular, respectively. Beam pointing variations caused by small wobble errors upon rotation are minimized by aligning one of the four mirrors. They are typically $<10 \mu \mathrm{rad}$ leading to a maximum beam shift of $15 \mu \mathrm{m}$ at the sample position. Note, in spectroscopy experiments, we keep the helicity constant and switch the magnetization direction with an electromagnet instead. This procedure leads to the same result as changing the helicity for a constant magnetization direction but is significantly faster. ${ }^{31}$ The principle of the four-mirror reflection phase shifter is extensively discussed in Refs. 21, 30, and 32.

\section{XUV intensity normalization}

Due to the non-linear high harmonic generation process, we observe intensity fluctuations across the generated XUV spectrum. Each orange diamond in Fig. 5 represents the normalized intensity of the 39th harmonic averaged over 2400 pulses $(0.8 \mathrm{~s}$ at $3 \mathrm{kHz})$. The data reveal fluctuations of $2.8 \% \mathrm{rms}$ from the HHG source, which are in the same order of magnitude as the expected MCD contrast, $A(E)$, of $1 \%-8 \% .{ }^{13}$ To resolve the pump induced changes of $A(E)$, we 


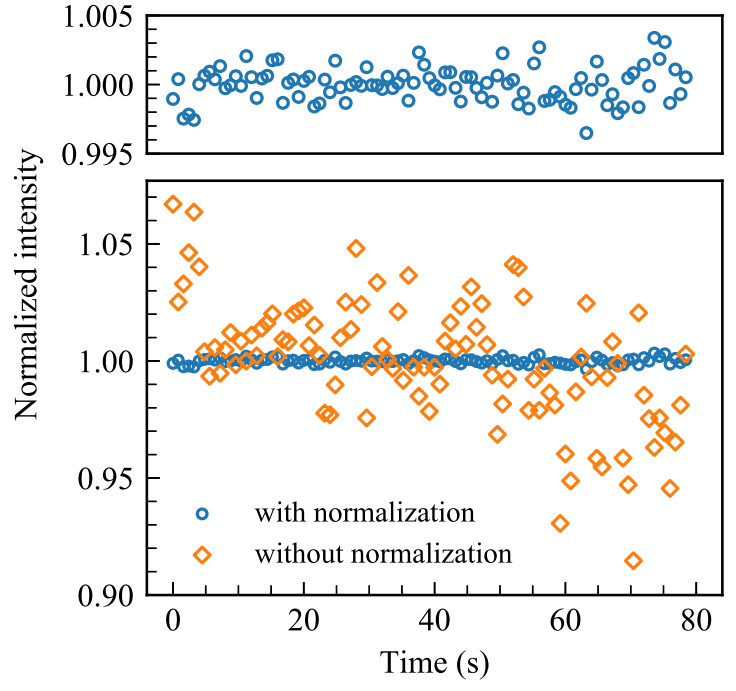

FIG. 5. Relative intensity fluctuations of one harmonic at $60 \mathrm{eV}$ as measured from the source (orange diamonds) and as normalized with the reference spectrometer (blue circles) as a function of time. The normalization scheme increases the signalto-noise ratio by at least one order of magnitude.

monitor the incident XUV spectrum with a reference spectrometer and use it to normalize the intensity measured with the spectrometer behind the sample. The blue circles in Fig. 5 show that the fluctuations are reduced to $0.12 \% \mathrm{rms}$ with this intensity normalization scheme. For measurements requiring long integration times, slower drifts are efficiently minimized as well.

The reference spectrometer consists of a plane grating, a wedge mirror, and a charged-coupled device (CCD). The grating with 2000 lines/mm and $15 \mathrm{~nm}-17 \mathrm{~nm}$ deep grooves (Eulitha AG, Switzerland) is coated with $23 \mathrm{~nm} \mathrm{~B}_{4} \mathrm{C}$ to reflect $\sim 70 \%$ of the light further towards the sample. Only $1 \%-2 \%$ of the incoming intensity gets diffracted into the first order and is then recorded with the CCD. Note that because the first order diffraction angle is only $2.3^{\circ}$ at $60 \mathrm{eV}$, we use a wedge mirror to increase the angle between the zeroth and first orders. To ensure an optimal energy resolution of the reference spectrometer, we performed a ray tracing calculation using RAY. ${ }^{33}$ This simulation helped us both to define the grating specification and to find the correct position of the grating and CCD. It allowed, furthermore, estimating the transmission of the optical elements. The spectral resolution at $60 \mathrm{eV}$ was calculated to be about $0.24 \mathrm{eV}$.

To achieve the best possible normalization, the number of photons on both cameras must be on the same order of magnitude. This ensures that the integration time is not limited by one of the cameras and that both cameras operate in the same dynamic range. Since the transmission through different samples varies, we compensate the intensity on the reference camera with $\mathrm{Al}$ filters of different thicknesses.

\section{Sample spectrometer}

The main spectrometer is located behind the sample in a separate vacuum chamber. It can be attached to the sample chamber in transmission or reflection geometry, depending on the experimental requirements. Here, we measure the entire spectrum that is either transmitted through or reflected off the sample. The focal spot with $\sim 150 \mu \mathrm{m}$ in diameter (FWHM) on the sample acts as our entrance slit. It is imaged by an aberration corrected, flat field concave grating (Hitachi, Japan) with 1200 lines/mm and a blaze angle $\Theta_{B}=3.7^{\circ}$ onto the CCD camera plane. From the parameters (focus size, grating parameters, and pixel size of the CCD), we calculate the spectrometer resolution to be $\sim 240 \mathrm{meV}$. From a measurement with a $20 \mu \mathrm{m}$ entrance slit, we determined the width of the high harmonic peaks to be $\sim 200 \mathrm{meV}$ (FWHM). An Al filter with a $200-\mathrm{nm}$ thickness at the entrance to the spectrometer vacuum chamber blocks the scattered IR pump light from reaching the detector.

We use identical cameras in both spectrometers (Greateyes $\mathrm{GmbH}$, Germany) with a chip geometry of 1024 pixels $\times 256$ pixels with pixel sizes of $26 \mu \mathrm{m} \times 26 \mu \mathrm{m}$. The energy axis $(1024$ pixels) is calibrated with the $\mathrm{Al} L$-edge at $72.55 \mathrm{eV}^{23}$ and the energy spacing between the individual HHG peaks of two times the fundamental driving energy. We obtain a spectral resolution of $25 \mathrm{meV} /$ pixel. To provide absolute photon counts, the cameras were calibrated by the National Metrology Institute of Germany (PTB) with the result of an energy-dependent conversion between 1.0 counts/photon and 3.1 counts/photon in the energy interval between $30 \mathrm{eV}$ and $95 \mathrm{eV}$ (cf. the Appendix for more information)

\section{The sample environment}

For time-resolved transmission experiments, the sample thickness is limited by the very short attenuation length of both the IR pump and XUV probe beams. To ensure a homogeneous excitation profile along the depth of the sample as well as a sufficient transmission of the XUV radiation, we typically use magnetic systems with a thickness between $10 \mathrm{~nm}$ and $20 \mathrm{~nm}$. Furthermore, the material studied has to be supported by a thin membrane: we commonly use either $20 \mathrm{~nm}$ thick $\mathrm{Si}_{3} \mathrm{~N}_{4}$ or a $100 \mathrm{~nm}$ thick $\mathrm{Al}$ foil in order to provide maximum XUV transparency and heat transport. To prevent oxidation, we deposit a capping layer onto the sample, usually $3 \mathrm{~nm}$ Pt or $4 \mathrm{~nm} \mathrm{Al}$.

The sample position relative to the pump and probe beams can be manipulated by piezo-activated translation stages (Smaract $\mathrm{GmbH}, \mathrm{Germany})$ in $x$-, $y$ - and $z$-directions with a precision of tens of nanometers. For MCD measurements of magnetic samples with in-plane magnetization, requiring a finite projection of the $\mathbf{k}$ vector of the probing light and the magnetization, the sample can be rotated around its axis, $\theta$. This also implies different magnet designs depending on the orientation of the easy axis of magnetization in the sample. For systems exhibiting out-of-plane magnetization, an electromagnet allows applying magnetic fields up to \pm 300 $\mathrm{mT}$, and for in-plane magnetization, the field is limited to $\approx \pm 40$ $\mathrm{mT}$ because of a larger gap between the iron poles. The electromagnet is controlled by a bipolar power supply (CAENels, FAST-Ps 1040-400), which allows toggling the magnetization with $10 \mathrm{~Hz}$. For temperature-dependent measurements between $10 \mathrm{~K}$ and $700 \mathrm{~K}$, a closed-cycle cryostat featuring ultra-low vibrations is available (Janis Research, USA). The cold finger can be manipulated in $x-, y$-, $z$ - and $\theta$-directions. 


\section{Time-resolved XUV spectroscopy}

Typically, we record the intensities of the entire spectrum of circularly polarized radiation transmitted through the sample for two opposite orientations of the applied magnetic field and, consequentially, orientations $( \pm)$ of the magnetization. This procedure corresponds to switching the helicity for a fixed magnetization direction. ${ }^{31}$ We repeat this measurement for all pump-probe delays and obtain the helicity-dependent transmission as a function of the delay time $t$ and photon energy $E, I(E, t)_{ \pm}$. The normalized difference between both measured intensities gives the magnetic asymmetry, $A(E, t)$, which under certain conditions is proportional to the magnetization of the sample, as discussed in Refs. 34 and 35,

$$
A(E, t)=\frac{I_{+}(E, t)-I_{-}(E, t)}{I_{+}(E, t)+I_{-}(E, t)} .
$$

Furthermore, the analysis of $I(E, t)_{+}$and $I(E, t)_{-}$, separately, gives us access to the helicity-dependent absorption, $\mu(E, t)_{ \pm}$ $=1-I(E, t)_{ \pm}$, assuming negligible reflectance in this wavelength range.

The time-resolved measurements are performed in an almost collinear pump-probe geometry. A beam splitter before the HHG apparatus generates a 5\% replica of the IR pulse used as the pump pulse. The pump pulse propagates over a linear translation stage, which controls the pump-probe delay. We control the pump intensity with a reflection-based attenuator consisting of a halfwave plate and two polarizers. The beam diameter at the sample position can be adjusted between $200 \mu \mathrm{m}$ and $900 \mu \mathrm{m}$ by controlling the distance of two lenses forming a telescope $(f=200 \mathrm{~mm}$ and $f=-100 \mathrm{~mm})$. To minimize the heat deposited in our sample system, we typically limit the pump spot size diameters to $500 \mu \mathrm{m}$ (FWHM).

We determine the spatial overlap of the pump and probe beams by directing each beam through a pinhole in the sample plane.

To determine the temporal overlap, we remove the Al filters and overlap both IR pulses-pump and remaining driving IR pulses-at the sample position. Here, we detect either the sumfrequency generation (SFG) signal from a barium borate (BBO) crystal or interference fringes on a beam profiler. The latter moreover demonstrates high phase stability between both beams. The temporal resolution of our pump-probe experiment is determined by measuring the SFG as a function of pump-probe delay. This cross correlation of the IR pulses corresponds to an upper limit for the temporal resolution, as the XUV pulses are significantly shorter than the driving IR pulses due to the HHG process. We can fit the SFG signal with a Gaussian function and obtain a FWHM of $46 \pm 3$ fs. To achieve this temporal resolution, we added a pair of chirped mirrors in the pump arm to compensate the additional dispersion caused by propagation through the air and transmission optics.

To demonstrate the performance of the spectrometer beamline, we performed helicity-dependent transient absorption experiments and investigated the magnetic asymmetry, $A(E, t)$, of a $15-\mathrm{nm}$ thick Co film.

Figure 6(a) shows the pump induced helicity-dependent transient absorption changes for two harmonic peaks with photon energies of $60.3 \mathrm{eV}$ and $69.6 \mathrm{eV}$. The pump fluence was set to $F_{\text {avg }}$ $=12 \mathrm{~mJ} / \mathrm{cm}^{-2}$. At the $3 p$ resonance of Co at $60.3 \mathrm{eV}$, we observe complicated dynamics, with increasing and decreasing absorption

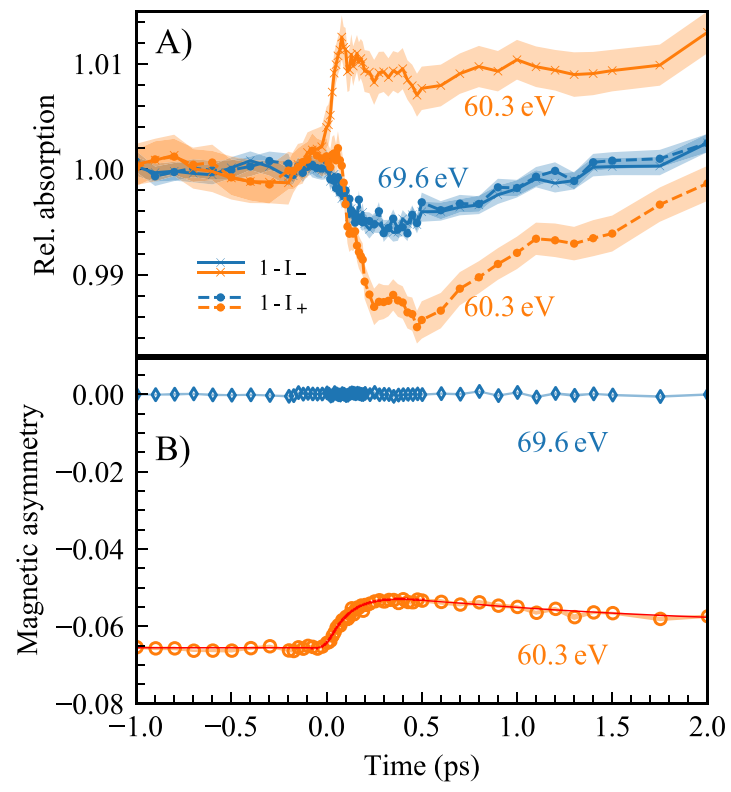

FIG. 6. (a) Relative changes of absorption in Co for two photon energies at $60.3 \mathrm{eV}$ (orange) and at $69.6 \mathrm{eV}$ (blue). (b) Magnetic asymmetry given by Eq. (1) as a function of pump-probe delay for on and off resonant photon energies. A double exponential fit yields a demagnetization time constant of $\tau=(124 \pm 4)$ fs. The shaded areas behind the data points represent the standard errors.

depending on the helicity of the XUV radiation. In a joint theoretical and experimental study, we were able to show that the measured transient absorption directly relates to the relative changes in the occupation of minority and majority states around the Fermi level. ${ }^{20}$ The reduced absorption above the resonance at $69.6 \mathrm{eV}$ can be explained by a spin-independent filling of higher energy states after the intense laser excitation. The possibility to monitor the spinresolved density of available states adds a significant additional value to our laboratory setup.

In Fig. 6(b), we show the normalized asymmetry, $A(E, t)$, defined by Eq. (1) as a function of the pump-probe delay. The magnetic asymmetry in the equilibrium state amounts to $6.5 \%$ at the resonance $(60.3 \mathrm{eV})$. After optical excitation, the asymmetry is quenched by $\sim 20 \%$ with a demagnetization time constant of $\tau$ $=(124 \pm 4) \mathrm{fs}$, revealed by a double exponential fit through the data points. At the off-resonant photon energy of $69.6 \mathrm{eV}$, we observe a constant asymmetry of zero. Note that the curves shown in Fig. 6 were averaged over 50 pump-probe delay scans. The effective integration time for each pump-probe delay point in panel (b) amounts to $1 \mathrm{~min}$, which corresponds to 50 repetitions and $600 \mathrm{~ms}$ acquisition time for two magnetization directions. The total measurement time was about five hours. After 50 repetitions, the standard error amounts to $\sim 5 \times 10^{-4}$ and is represented by the shaded area behind the data points.

\section{XUV scattering instrument}

Given the XUV wavelength of about $15 \mathrm{~nm}-30 \mathrm{~nm}$, scattering experiments can additionally provide nanometer-scale spatial 
information of the excited magnetic system. As these experiments typically require monochromatic radiation, they particularly benefit from a high peak flux and small bandwidths of the harmonics. Sharing the same XUV source with the spectroscopy beamline allows optimizing the HHG parameters in this respect. After optimization, the motorized SMU is removed from the beam path, and the direct beam of the HHG source reaches the scattering instrument (Fig. 1).

We designed the vacuum chamber with a rectangular invacuum optical breadboard of $500 \mathrm{~mm} \times 800 \mathrm{~mm}$ size, providing high flexibility for the experimental geometry. Several stepper-motor (Standa Ltd., Lithuania) and piezo-driven (SmarAct GmbH, Germany) stages can be freely arranged for the manipulation and positioning of the optics, sample, and detector. In the standard configuration, as shown in Fig. 1, a spherical multilayer mirror selects a single harmonic peak and focuses the XUV beam under nearly normal incidence. The multilayer design is specifically adapted to the harmonic required for the experiment. A measurement by PTB of the multilayer mirror (optiX fab $\mathrm{GmbH}$, Germany) selecting the 39th harmonic determined a reflectivity of $51 \%$ at $60.8 \mathrm{eV}$ with a bandwidth of $2.1 \mathrm{eV}$ (FWHM). The neighboring harmonics are suppressed at a ratio of 1:17. An additional plane multilayer mirror is inserted if a higher purity is needed. The beam is focused to a minimum spot size on the sample of $10 \mu \mathrm{m} \times 10 \mu \mathrm{m}$ (FWHM). A back-illuminated in-vacuum CCD camera with 2048 pixels $\times 2048$ pixels of $13.5 \mu \mathrm{m}$ pixel size (GE-VAC, Greateyes GmbH, Germany) detects the radiation scattered from the sample. An Al filter (Luxel, USA) is mounted on the camera and stops visible and IR pump light. A beamstop blocks the intense forward scattered XUV radiation. For time-resolved pump-probe experiments, the IR pump is derived from the same beam used for the spectroscopy experiment, thus sharing the intensity and pump-probe delay control as well as pointing stabilization (Fig. 1). A long-distance microscope (DistaMax K2, Infinity Photo-Optical Company, USA) allows us to precisely monitor the position of the sample as well as the size and position of the pump beam on the sample. A compact in-vacuum electromagnet is available to apply fields of up to $350 \mathrm{mT}$ at the sample in the out-of-plane direction.

Figure 7(a) shows a result from a typical small-angle resonant scattering experiment where we detect the scattering from magnetic domains in a $[\mathrm{Co}(0.8) / \mathrm{Pt}(0.8)]_{16}$ multilayer. In this sample, domains of antiparallel out-of-plane magnetization with $\sim 180 \mathrm{~nm}$ width form a labyrinth-like pattern in the remanent state due to the uniaxial perpendicular magnetic anisotropy of the multilayer. ${ }^{36}$ When selecting an XUV photon energy close to the $M_{2,3}$ absorption edge of $\mathrm{Co}$, the MCD in the photo-absorption in the oppositely magnetized domains leads to the characteristic ring-like scattering pattern. The anisotropic intensity distribution indicates a preferred alignment of the domains. While the size and shape of the scattering ring provide direct information on the domain's average width, size distribution, and orientation, the scattering intensity is proportional to the square of the magnetization within each domain. ${ }^{37}$ XUV scattering methods, thus, provide background-free, element-specific access to the magnitude and spatial distribution of magnetization in the thin film. ${ }^{12,}$

In Fig. 7(b), we show the relative changes of the magnetization after the sample was excited by the IR laser. The red frames in Fig. 7(a) indicate the integration areas. Similarly to the MCD results
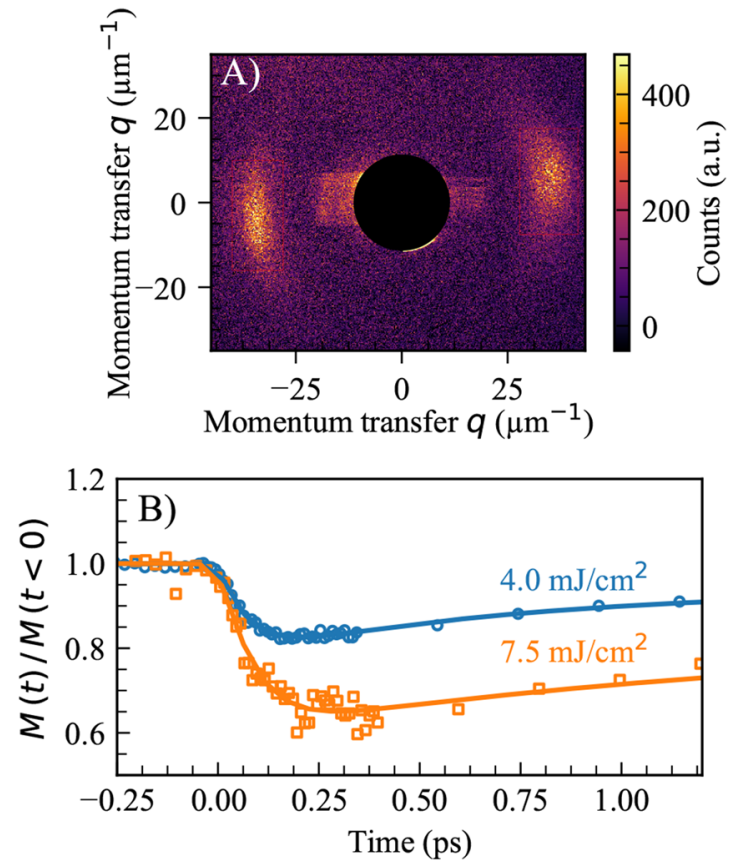

FIG. 7. (a) Resonant small-angle scattering from magnetic domains of a Co/Pt multilayer sample on the Co $\mathrm{M}_{2,3}$-edge in transmission. The intensity of the scattering ring is proportional to the magnetization squared. (b) Relative magnetization as a function of pump-probe delay for two excitation fluences. The red frames in (a) represent the integration areas.

shown in Fig. 6(b), we observe an ultrafast demagnetization of the excited $\mathrm{Co} / \mathrm{Pt}$ film followed by a slower recovery. ${ }^{12,38}$

\section{CONCLUSIONS}

We demonstrated the performance of a laboratory based high harmonic driven experimental setup combining two instruments to measure spin and electron dynamics with femtosecond temporal and nanometer spatial resolution. With the spectroscopy instrument, we can perform energy-resolved and polarization-dependent experiments in both transmission and reflection geometries. With the second instrument, we conduct time-resolved small-angle scattering experiments. Both instruments use the XUV radiation generated from our HHG source reaching a calibrated photon flux of about $(3 \pm 1) \times 10^{10}$ photons/s/harmonic at $60 \mathrm{eV}$. A fourmirror reflection phase shifter enables controlling the XUV polarization between linear to circular, allowing us to measure the XUV MCD contrast in magnetic materials. The spectral normalization scheme reduces the intensity fluctuations to $0.12 \% \mathrm{rms}$ enabling the detection of small pump induced effects. We performed timeresolved measurements in a pump-probe geometry to determine the ultrafast magnetization dynamics via MCD for a Co film and via magnetic small-angle scattering from magnetic domains of $\mathrm{Co} / \mathrm{Pt}$ multilayers. The possibility to extract details of the spin-dependent non-equilibrium electronic structure via helicity-dependent transient absorption allows disentangling the complex physics of spin excitations in functional magnetic systems. 


\section{ACKNOWLEDGMENTS}

C.v.K.S. and S.E. would like to thank DFG for funding through TRR227 Project No. A02. The authors acknowledge helpful discussions with Boris Vodungbo regarding the design of the XUV/IR beam splitter. F.W. and C.v.K.S. would like to thank Oleg Kornilov and Nick Zhavoronkov for sharing their expertise regarding high harmonic generation.

\section{APPENDIX: DETERMINATION OF THE ABSOLUTE HHC PHOTON FLUX}

The determination of an absolute number of the photon flux relies on a calibrated detector and on accurate knowledge about the reflectance of beamline optics. For this reason, precise measurements of the efficiency of our detector and our XUV mirrors were performed at the Metrology Light Source of the PTB in Berlin, Germany. The detection efficiency of the CCD camera (Greateyes GE 1024256 BI UV1), i.e., the number of counts per incident photon, is shown in Fig. 8(a) as a function of the photon energy. It was measured in the maximum dynamic range-mode. In Fig. 8(b), we show the reflectance of an XUV mirror with a $\mathrm{B}_{4} \mathrm{C}$ coating with $23 \mathrm{~nm}$ thickness for grazing incidence angles of $4^{\circ}$ and $5^{\circ}$ as a function of energy. The absorption of the Al filters is measured with the spectroscopy camera behind the sample. We use calculated values for the
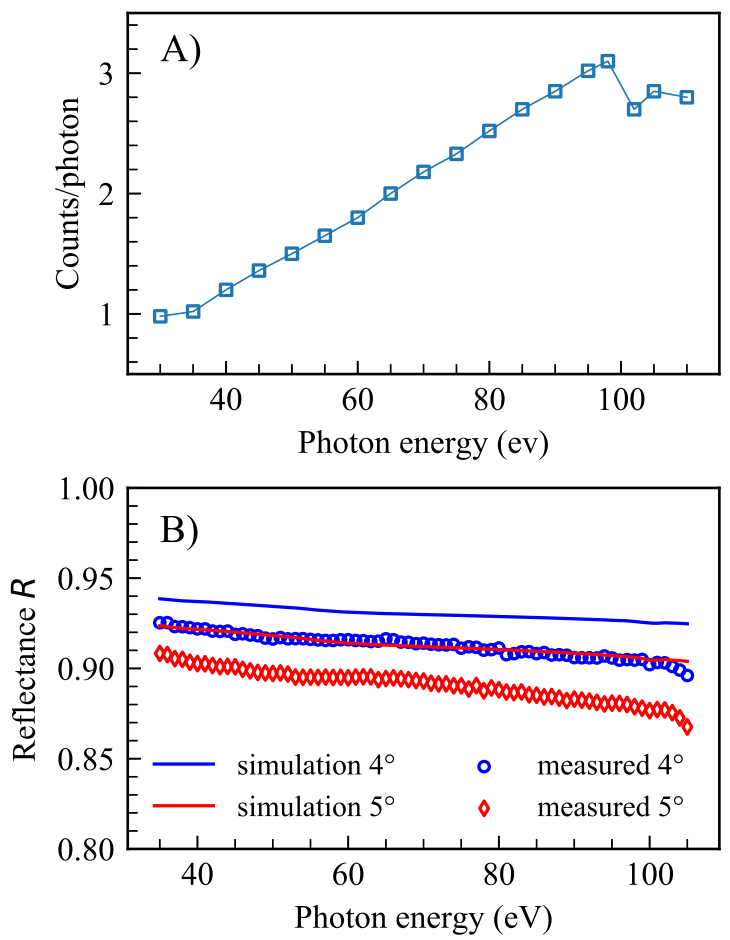

FIG. 8. (a) The calibration curve for the Greateyes CCD cameras (GE1024 $256 \mathrm{BI}$ UV1) shows counts per photon as a function of the photon energy. (b) Reflectance of the XUV mirrors with a 23-nm thick $\mathrm{B}_{4} \mathrm{C}$ coating for grazing incident angles of $4^{\circ}$ and $5^{\circ}$ as a function of the photon energy. Both measurements were performed at the Metrology Light Source of PTB, Berlin.
TABLE I. XUV transmission of all the optical elements from the HHG source to the camera behind the sample in the photon energy range from $45 \mathrm{eV}$ to $72 \mathrm{eV}$. The angles for reflective optics are given as grazing incidence angles. The values have been measured unless otherwise indicated.

Element XUV transmission in \% Error in \%

XUV/IR splitter $\left(7^{\circ}\right)$

$\operatorname{SMU}\left(4^{\circ}\right)$

Toroid $1\left(5^{\circ}\right)$

$69-52 \quad \pm 2$

Phase shifter

90

92

Al filter

Reference grating $\left(1^{\text {st }}\right.$ order$)$

Reference grating $\left(0^{\text {th }}\right.$ order $)$

Toroid $2\left(5^{\circ}\right)$

Al filter

Grating behind the sample

Total on spectroscopy camera

$10-32$

$\pm 2$

39-54

$\pm 3.3$

13-18 (calculated) $\quad \pm 3$

70 (calculated) $\quad \pm 5$

$92 \pm 2$

$39-54 \quad \pm 1.5$

13-18 (calculated) $\quad \pm 3$

$0.07-0.47 \quad \pm 0.01$

efficiency of the flat-field dispersion grating (Hitachi, part no. 0010640) ranging between $13 \%$ and $18 \%$ for the relevant energy range. However, we have confirmed for similar gratings (Hitachi, part no. 001-0437) that the calculated and measured values only deviate by a few percent.

The transmission of all the optical elements in the spectral range from $45 \mathrm{eV}$ to $72 \mathrm{eV}$ is listed in Table I. From the HHG source, $(0.04 \%-0.47 \%)$ of the photons with energies from $45 \mathrm{eV}$ to $72 \mathrm{eV}$ reach the camera behind the sample.

\section{DATA AVAILABILITY}

The data that support the findings of this study are available from the corresponding author upon reasonable request.

\section{REFERENCES}

${ }^{1}$ E. Beaurepaire, J.-C. Merle, A. Daunois, and J.-Y. Bigot, Phys. Rev. Lett. 76, 4250 (1996).

${ }^{2}$ C. D. Stanciu, F. Hansteen, A. V. Kimel, A. Kirilyuk, A. Tsukamoto, A. Itoh, and T. Rasing, Phys. Rev. Lett. 99, 217204 (2007).

${ }^{3}$ I. Radu, K. Vahaplar, C. Stamm, T. Kachel, N. Pontius, H. A. Dürr, T. A. Ostler, J. Barker, R. F. L. Evans, R. W. Chantrell, A. Tsukamoto, A. Itoh, A. Kirilyuk, T. Rasing, and A. V. Kimel, Nature 472, 205 (2011).

${ }^{4}$ T. Kampfrath, M. Battiato, P. Maldonado, G. Eilers, J. Nötzold, S. Mährlein, V. Zbarsky, F. Freimuth, Y. Mokrousov, S. Blügel, M. Wolf, I. Radu, P. M. Oppeneer, and M. Münzenberg, Nat. Nanotechnol. 8, 256 (2013).

${ }^{5}$ T. Seifert, U. Martens, S. Günther, M. A. W. Schoen, F. Radu, X. Z. Chen, I. Lucas, R. Ramos, M. H. Aguirre, P. A. Algarabel, A. Anadón, H. S. Körner, J. Walowski, C. Back, M. R. Ibarra, L. Morellón, E. Saitoh, M. Wolf, C. Song, K. Uchida, M. Münzenberg, I. Radu, and T. Kampfrath, SPIN 07, 1740010 (2017); arXiv:1705.11069.

${ }^{6}$ J. K. Dewhurst, P. Elliott, S. Shallcross, E. K. U. Gross, and S. Sharma, Nano Lett. 18, 1842 (2018).

${ }^{7}$ C. Stamm, T. Kachel, N. Pontius, R. Mitzner, T. Quast, K. Holldack, S. Khan, C. Lupulescu, E. F. Aziz, M. Wietstruk, H. A. Dürr, and W. Eberhardt, Nat. Mater. 6, 740 (2007)

${ }^{8}$ K. Holldack, J. Bahrdt, A. Balzer, U. Bovensiepen, M. Brzhezinskaya, A. Erko, A. Eschenlohr, R. Follath, A. Firsov, W. Frentrup, L. Le Guyader, T. Kachel,

P. Kuske, R. Mitzner, R. Müller, N. Pontius, T. Quast, I. Radu, J.-S. Schmidt, 
C. Schüßler-Langeheine, M. Sperling, C. Stamm, C. Trabant, and A. Föhlisch, J. Synchrotron Radiat. 21, 1090 (2014).

${ }^{9}$ D. J. Higley, K. Hirsch, G. L. Dakovski, E. Jal, E. Yuan, T. Liu, A. A. Lutman, J. P. Macarthur, E. Arenholz, Z. Chen, G. Coslovich, P. Denes, P. W. Granitzka, P. Hart, M. C. Hoffmann, J. Joseph, L. Le Guyader, A. Mitra, S. Moeller, H. Ohldag, M. Seaberg, P. Shafer, J. Stöhr, A. Tsukamoto, H.-D. Nuhn, A. H. Reid, H. A. Dürr, and W. F. Schlotter, Rev. Sci. Instrum. 87, 033110 (2016); arXiv:1511.07372.

${ }^{10}$ F. Capotondi, E. Pedersoli, N. Mahne, R. H. Menk, G. Passos, L. Raimondi, C. Svetina, G. Sandrin, M. Zangrando, M. Kiskinova, S. Bajt, M. Barthelmess, H. Fleckenstein, H. N. Chapman, J. Schulz, J. Bach, R. Frömter, S. Schleitzer, L. Müller, C. Gutt, and G. Grübel, Rev. Sci. Instrum. 84, 051301 (2013).

${ }^{11}$ C. La-O-Vorakiat, M. Siemens, M. M. Murnane, H. C. Kapteyn, S. Mathias, M. Aeschlimann, P. Grychtol, R. Adam, C. M. Schneider, J. M. Shaw, H. Nembach, and T. J. Silva, Phys. Rev. Lett. 103, 257402 (2009).

${ }^{12}$ B. Vodungbo, J. Gautier, G. Lambert, A. B. Sardinha, M. Lozano, S. Sebban, M. Ducousso, W. Boutu, K. Li, B. Tudu, M. Tortarolo, R. Hawaldar, R. Delaunay, V. López-Flores, J. Arabski, C. Boeglin, H. Merdji, P. Zeitoun, and J. Lüning, Nat. Commun. 3, 999 (2012).

${ }^{13}$ F. Willems, C. T. L. Smeenk, N. Zhavoronkov, O. Kornilov, I. Radu, M. Schmidbauer, M. Hanke, C. von Korff Schmising, M. J. J. Vrakking, and S. Eisebitt, Phys. Rev. B 92, 220405 (2015).

${ }^{14}$ C. La-O-Vorakiat, E. Turgut, C. A. Teale, H. C. Kapteyn, M. M. Murnane, S. Mathias, M. Aeschlimann, C. M. Schneider, J. M. Shaw, H. T. Nembach, and T. J. Silva, Phys. Rev. X 2, 011005 (2012).

${ }^{15}$ S. Jana, J. A. Terschlüsen, R. Stefanuik, S. Plogmaker, S. Troisi, R. S. Malik, M. Svanqvist, R. Knut, J. Söderström, and O. Karis, Rev. Sci. Instrum. 88, 033113 (2017).

${ }^{16}$ M. Hecker, P. M. Oppeneer, S. Valencia, H.-C. Mertins, and C. M. Schneider, J. Electron Spectrosc. Relat. Phenom. 144-147, 881 (2005).

${ }^{17}$ F. Siegrist, J. A. Gessner, M. Ossiander, C. Denker, Y.-P. Chang, M. C. Schröder, A. Guggenmos, Y. Cui, J. Walowski, U. Martens, J. K. Dewhurst, U. Kleineberg, M. Münzenberg, S. Sharma, and M. Schultze, Nature 571, 240 (2019).

${ }^{18}$ C. Alves, G. Lambert, V. Malka, M. Hehn, G. Malinowski, M. Hennes, V. Chardonnet, E. Jal, J. Lüning, and B. Vodungbo, Phys. Rev. B 100, 144421 (2019).

${ }^{19}$ F. Willems, S. Sharma, C. v. Korff Schmising, J. K. Dewhurst, L. Salemi, D. Schick, P. Hessing, C. Strüber, W. D. Engel, and S. Eisebitt, Phys. Rev. Lett. 122, 217202 (2019); arXiv:1812.06703.

${ }^{20}$ F. Willems, C. von Korff Schmising, C. Strüber, D. Schick, D. W. Engel, J. K. Dewhurst, P. Elliott, S. Sharma, and S. Eisebitt, Nat. Commun. 11, 871 (2020).
${ }^{21}$ C. von Korff Schmising, D. Weder, T. Noll, B. Pfau, M. Hennecke, C. Strüber, I. Radu, M. Schneider, S. Staeck, C. M. Günther, J. Lüning, A. E. D. Merhe, J. Buck, G. Hartmann, J. Viefhaus, R. Treusch, and S. Eisebitt, Rev. Sci. Instrum. 88, 053903 (2017).

$\mathbf{2 2}_{\text {https://sardana-controls.org/. }}$

${ }^{23}$ J. A. Bearden and A. F. Burr, Rev. Mod. Phys. 39, 125 (1967).

${ }^{24}$ S. Kazamias, F. Weihe, D. Douillet, C. Valentin, T. Planchon, S. Sebban, G. Grillon, F. Augé, D. Hulin, and P. Balcou, Eur. Phys. J. D 21, 353 (2002).

${ }^{25}$ W. Becker, S. Long, and J. K. McIver, Phys. Rev. A 41, 4112 (1990).

${ }^{26}$ M. Lewenstein, P. Balcou, M. Y. Ivanov, A. L'Huillier, and P. B. Corkum, Phys. Rev. A 49, 2117 (1994).

${ }^{27}$ B. L. Henke, E. M. Gullikson, and J. C. Davis, At. Data Nucl. Data Tables 54, 181 (1993).

${ }^{28}$ Handbook of Optical Constants of Solids, edited by E. Palik (Academic Press, $1985)$.

${ }^{29}$ D. L. Windt, Appl. Opt. 30, 15 (1991).

${ }^{30}$ B. Vodungbo, A. Barszczak Sardinha, J. Gautier, G. Lambert, C. Valentin, M. Lozano, G. Iaquaniello, F. Delmotte, S. Sebban, J. Lüning, and P. Zeitoun, Opt. Express 19, 4346 (2011).

${ }^{31} \mathrm{~J}$. Stöhr and Y. Wu, New Directions in Research with Third-Generation Soft XRay Synchrotron Radiation Sources (Springer Netherlands, Dordrecht, 1994), pp. 221-250.

${ }^{32}$ H. Höchst, D. Zhao, and D. L. Huber, Surf. Sci. 352-354, 998 (1996).

${ }^{33}$ F. Schäfers, in Modern Developments in X-Ray and Neutron Optics, Springer Series in optical science Vol. 137, edited by A. Erko, M. Idir, T. Krist, and A. G. Michette (Springer Berlin Heidelberg, Berlin, Heidelberg, 2008), pp. 9-41.

${ }^{34}$ J. K. Dewhurst, F. Willems, P. Elliott, Q. Z. Li, C. V. K. Schmising, C. Strüber, D. W. Engel, S. Eisebitt, and S. Sharma, Phys. Rev. Lett. 124, 077203 (2020); arXiv:1909.00199.

${ }^{35}$ K. Yao, F. Willems, C. v. K. Schmising, I. Radu, C. Strüber, D. Schick, D. Engel, A. Tsukamoto, J. K. Dewhurst, S. Sharma, and S. Eisebitt, Phys. Rev. B 102, 100405(R) (2020)

${ }^{36}$ O. Hellwig, A. Berger, J. B. Kortright, and E. E. Fullerton, J. Magn. Magn. Mater. 319, 13 (2007).

${ }^{37}$ J. B. Kortright, J. Electron Spectrosc. Relat. Phenom. 189, 178 (2013).

${ }^{38}$ B. Pfau, S. Schaffert, L. Müller, C. Gutt, A. Al-Shemmary, F. Büttner, R. Delaunay, S. Düsterer, S. Flewett, R. Frömter, J. Geilhufe, E. Guehrs, C. Günther, R. Hawaldar, M. Hille, N. Jaouen, A. Kobs, K. Li, J. Mohanty, H. Redlin, W. Schlotter, D. Stickler, R. Treusch, B. Vodungbo, M. Kläui, H. Oepen, J. Lüning, G. Grübel, and S. Eisebitt, Nat. Commun. 3, 1100 (2012). 